\title{
Reference-Driven Compressed Sensing MR Image Reconstruction with Partially Known Support and Group Sparsity Constraints
}

\author{
Yize Dong, Huiqian Du, Di Zhao, Yu Han, Wenbo Mei
}

\begin{abstract}
Applying compressed sensing (CS) to magnetic resonance imaging (MRI) makes it possible to reconstruct a MR image from undersampled data. Traditional CS based MR image reconstruction schemes only use the signals' sparsity in an appropriate transform domain to reduce sampling rate. This paper proposes a new MR image reconstruction method which utilizes structure features of the image besides sparsity. The proposed method exploits the distribution of the wavelet coefficients' magnitudes and support information to formulate the objective function by minimizing which the image is reconstructed. The objective function consists of the data consistence term and two regularization terms: $l_{1}-l_{2}$ norm of the groups which contains the coefficients with the unknown support and total variation (TV) norm of the image. The simulation results from real MR images show that the proposed method outperforms the conventional CS based MR image reconstruction method.
\end{abstract}

Keywords: Compressed Sensing - MR image reconstruction - The reference image $\cdot$ Support $\cdot$ Group sparsity

\section{Introduction}

Accelerating the speed of MRI has always been the research focus in MRI research community. CS-based methods make it possible to reconstruct MR image from undersampled data by exploiting the image's sparsity, which is known as CS-MRI [3, 4, 11]. In nowadays, many researchers hope to exploit prior information besides sparsity to further decrease the sampling rate. Lu and Do [6]

\footnotetext{
${ }^{1}$ Huiqian Du $(\bowtie)$

Beijing Institute of Technology

Beijing, China

e-mail: duhuiqian@bit.edu.cn
}

Yize Dong, Di Zhao, Yu Han, Weibo Mei

Beijing Institute of Technology

Beijing, China 
presented a theory for sampling signals from a union of subspaces. This theory can be utilized to analyze the structure features of signals and it provides a path for extending the current CS from finite dimensional cases to infinite-dimensional cases. Vaswani and $\mathrm{Lu}$ et al. $[5,9,13]$ proposed a modified-CS model which improves the quality of the reconstructed image dramatically by only penalizing the coefficients with unknown support. Baraniuk [2] proposed a Model-based CS, which exploits the inherent tree structure of the wavelet coefficients except for sparsity. Stojnic, Parvaresh and Hassibi [10] proposed a block sparsity model for high probability recovery of the block-sparse signals. Since Lustig et al. [7] proposed CS-based MR image reconstruction, applying state-of-the-art methods in CS to MRI reconstruction is one of the CS-MRI research trends. Prieto and Usman et al. $[8,12]$ improved the standard CS-MRI by exploiting the group sparsity of dynamic MRI. Babacan et al. [1] presented a framework based on image modeling within union-of-subspaces, and applying it to interventional MRI (iMRI).

In this paper, we intend to utilize both group sparisity and support information of the wavelet coefficients to further decrease the sampling rate for CS-MRI. In many MRI applications, a high-resolution reference image with similar contents to the target image can be easily acquired. Since the distribution of wavelet coefficients of the target image is similar to that of the reference image, we proposed to get the support and grouping information of the wavelet coefficients of the target image according to the reference image. The reconstruction is formulated as an optimization problem where the objective function consists of a data consistence term and two regularization terms, one is the $l_{1}-l_{2}$ norm of the groups which contains the coefficients with the unknown support, and the other is TV norm of the image. Minimizing the $l_{1}-l_{2}$ norm of the groups with unknown support will enhance the group sparsity and transit the support information to the reconstruction result. We evaluate the performance of the proposed method by simulations based on real MR images and compare our method with the conventional CS-MRI method [7]. The results prove that the proposed method is able to reconstruct high quality MR image from fewer sampling data.

\section{Reconstruction Model of Conventional CS-MRI}

In the MRI, the data acquisition is formulated as:

$$
\mathbf{y}=\mathbf{F}_{\mathbf{u}} \mathbf{I}_{\mathbf{t}}+\mathbf{n}
$$

where $\mathbf{y}$ is the measured data vector of the target image $\mathbf{I}_{\mathbf{t}}$ and $\mathbf{F}_{\mathbf{u}}$ is an undersampled Fourier transform operator, $\mathbf{n}$ is the measurement noise.

The target image can be exactly reconstructed by solving the following optimization problem according to CS theory: 


$$
\hat{\mathbf{I}}_{\mathbf{t}}=\min \arg \left\|\mathbf{y}-\mathbf{F}_{\mathbf{u}} \mathbf{I}_{\mathbf{t}}\right\|_{2}^{2}+l_{1}\left\|\mathbf{\Psi} \mathbf{I}_{\mathbf{t}}\right\|_{1}+l_{2}\left\|\mathbf{I}_{\mathbf{t}}\right\|_{T V}
$$

where $\hat{\mathbf{I}}_{\mathbf{t}}$ denotes the estimation for $\mathbf{I}_{\mathbf{t}} \cdot\|\|_{T V}$ is the TV operator, defined as $\|\mathbf{x}\|_{T V}=\sum_{i, j}\left(\left|\left(D_{h} x\right)_{i, j}\right|+\left|\left(D_{v} x\right)_{i, j}\right|\right)$ in this paper, and $\left(D_{h} x\right)_{i, j}=x_{i+1, j}-x_{i, j},\left(D_{v} x\right)_{i, j}=x_{i, j+1}-x_{i, j}$.

The linear operator $\boldsymbol{\Psi}$ is the wavelet transform. The regularization parameters $l_{1}, l_{2}$ are positive constants.

\section{Proposed Method}

We exploit the distribution of the wavelet coefficients' magnitudes and support information. The image reconstruction problem is formulated as minimizing the objective function which comprises the prior information besides sparsity.

\subsection{Structure Similarity between the Reference and the Target Image}

To describe our method clearly, we compare the similarity of the wavelet coefficients' distribution between the reference and the target image. We chose images shown in Fig.1 (A) and Fig.1 (B) as the reference image and the target image. The wavelet coefficients of two images are displayed in Fig. 2, where a 4 level Daubechies-2 wavelet transform is performed on them. For simplicity, we will simplify the wavelet coefficients of the target image into the target coefficients, and the wavelet coefficients of the reference image into the reference coefficients in the rest of the paper.

As we expect the anatomical structure observed in the reference image appears at the similar support in the target image. The support of both images is generally consistent, and the magnitude distribution is similar too. The group sparsity exists if we divide the reference coefficients with close magnitude into groups. Hence it is reasonable to guess the support of the target coefficients based on that of the reference coefficients. If we hope to make full use of structure feature similarity, we may divide the target wavelet coefficients into disjoint groups in the same way that the reference coefficients are divided. 


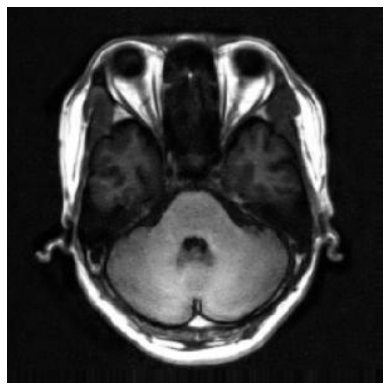

A

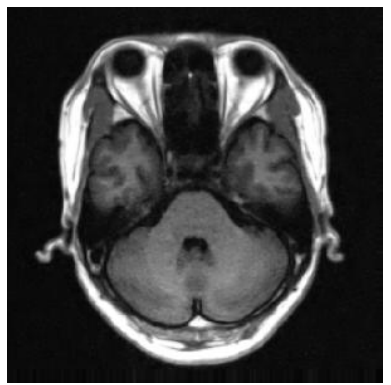

B

Fig. 1 The reference image (A) and the target image (B)

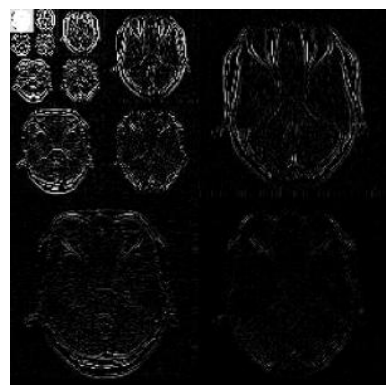

A

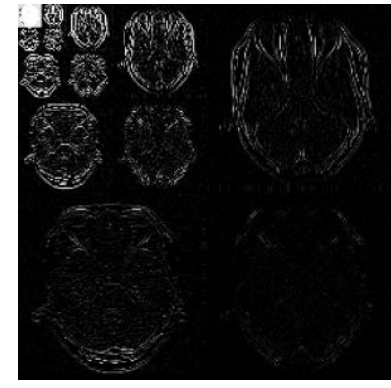

B

Fig. 2 The wavelet coefficients matrices of the reference image (A) and the target image (B)

\subsection{Proposed Method}

From the modified-CS model, we know that when a part of the signal support is known, constraining the part with unknown support will improve the reconstruction performance quite well. In addition, if a signal has group sparsity, the required sampling rate will decrease too. Based on the above theory and analysis, we propose to seek the target image with sparse groups and known support, which also agrees with the measurement $y$. The reconstruction is obtained by solving the following constrained optimization problem:

$$
\hat{\mathbf{I}}_{\mathbf{t}}=\underset{\mathbf{I}_{\mathbf{t}}}{\min \arg }\left\|\mathbf{y}-\mathbf{F}_{\mathbf{u}} \mathbf{I}_{\mathbf{t}}\right\|_{2}^{2}+l_{1}\left\|\left(\mathbf{\Psi I}_{\mathbf{t}}\right)_{T_{r}^{c}}^{(g)}\right\|_{1,2}+l_{2}\left\|\mathbf{I}_{\mathbf{t}}\right\|_{T V}
$$

the first term of the objective function in Eq. (3) enforces data consistency. The second term is a $l_{1}-l_{2}$ mixed norm. Minimizing $\left\|\left(\mathbf{\Psi}_{\mathbf{t}}\right)_{T_{r}^{c}}^{(g)}\right\|_{1,2}$ promotes group sparsity.

The third term is induced to promote the sparsity in the discrete gradient domain. 
The constants $l_{1}, l_{2}$ are used to adjust the weights of the second and the third term. In the following, we will specify the second term.

Assume the reference image and the target image have the size of $m * n$, and $M=m * n \cdot T_{r}$ is a set containing the known part of the support of the reference coefficients and $T_{r}^{c}=[1: M] \backslash T_{r}$ denotes the complement of $T_{r}$. The term $\left(\mathbf{\Psi I}_{\mathbf{t}}\right)_{T_{r}^{c}}$ denotes the partial target coefficients whose support belonging to $T_{r}^{c}$ set. The term $\left\|\left(\boldsymbol{\Psi}_{\mathbf{t}}\right)_{T_{r}}^{(g)}\right\|_{1,2} \|_{1,2}$ is defined as the mixed $l_{1}-l_{2}$ norm of the wavelet coefficients which are divided into $K$ groups,

$$
\left\|\left(\mathbf{\Psi I}_{\mathbf{t}}\right)_{T_{r}^{c}}^{(g)}\right\|_{1,2}=\left\|\mathbf{W}_{\mathbf{c}}^{(g)}\right\|_{1,2}=\left\|\mathbf{W}_{\mathbf{c}}^{g_{1}}\right\|_{2}+\left\|\mathbf{W}_{\mathbf{c}}^{g_{2}}\right\|_{2}+\cdots+\left\|\mathbf{W}_{\mathbf{c}}^{g_{K}}\right\|_{2}
$$

where $\mathbf{W}_{\mathbf{c}}=\left(\boldsymbol{\Psi}_{\mathbf{t}}\right)_{T_{r}^{c}}$ for simplicity, and $\left\|\mathbf{W}_{\mathbf{c}}^{s i}\right\|_{2}$ is the $l_{2}$ norm of all wavelet coefficients assigned to the $i$ th group $g_{i}(i=1,2, \ldots, K)$. While $\bigcup_{i=1}^{K} g_{i}=T_{r}^{c}$, and $g_{i} \cap g_{j}=\varnothing(i \neq j)$.

\subsection{Method}

The proposed method comprises three steps: support acquisition, group assignment, and optimization algorithm.

\subsubsection{Support Acquisition}

We suggest to determine $T_{r}$ by setting a threshold $a$, that is

$$
T_{r}=\left\{i \in[1: M]:\left(\mathbf{\Psi I}_{\mathbf{r}}\right)_{i}^{2}>a\right\}
$$

usually $a$ is chosen to guarantee the reference coefficients whose support belonging to $T_{r}$ account for $99 \%$ of the entire image energy. The support of the target coefficients denoted as $S$, can be split as $S=\left(T_{r} \backslash \Delta_{d}\right) \cup \Delta, \Delta$ is the unknown part of the support in the target coefficients, and $\Delta_{d}$ is the error in the known support $T_{r}$ between the reference image and the target image. The set operation $U$ and $\backslash$ indicate the union and difference.

Another equivalent way to get $T_{r}$ is that arrange the reference coefficients in descending order, and select the support of the first $k$ coefficients to construct $T_{r}$. This procedure provides the same effect as using the threshold $a$, but is more maneuverable. 


\subsubsection{Group Assignment}

For the grouping information, we divide the reference coefficients with close magnitude into $K$ disjoint groups. The size of all the groups is the same which is denoted as $l$. Thus $\left|g_{i}\right|=l, \bigcup_{i=1}^{K} g_{i}=T_{r}^{c}$. The length of the unknown support $T_{r}^{c}$ must satisfy $\left|T_{r}^{c}\right|=l \times K$.

Due to the similarity between the reference and the target coefficients, we assume the target coefficients have the similar support and magnitude distribution as that of the reference image. Hence we divide the target coefficients whose support belongs to $T_{r}^{c}$ into groups according to the same grouping criterion as grouping the reference coefficients.

\subsubsection{Optimization Algorithm}

After group assignment, the target image is reconstructed by solving the formulation given in Eq. (3) using a nonlinear conjugate gradient reconstruction algorithm.

\section{Experimental Results}

In this section, we evaluate the performance of our method using real MR images, with the size of $256 \times 256$. We used Fig. 1 (A) as the reference image and Fig. 1 (B) as the target image.

We retained the largest $10 \%$ reference coefficients (corresponding to $99 \%$ of the image energy) as the known part to make up of $T_{r}$. The rest coefficients determine $T_{r}^{c}$. For the group assignment, coefficients whose support belonging to $T_{r}^{c}$ were assigned to distinct groups according to their magnitudes as mentioned in section 3 .

All reconstructions were implemented in Matlab using the non-linear conjugate gradient algorithm. The images reconstructed by our method and convention CSMRI are shown in Fig. 3. Figure 4 (A) compares the relative errors of reconstruction by two methods. The curves characterize the relationship between reconstruction accuracy and the percentage of data acquired which also demonstrate our proposed method outperforms the conventional CS-MRI at any undersampling rate. As we know, the coefficients assigned to even groups with different sizes would influence the recovery performance. It shows that 5 is the optimal size of groups in Fig. 4 (B). With this optimal size, our method performs better with smallest recovery error. 


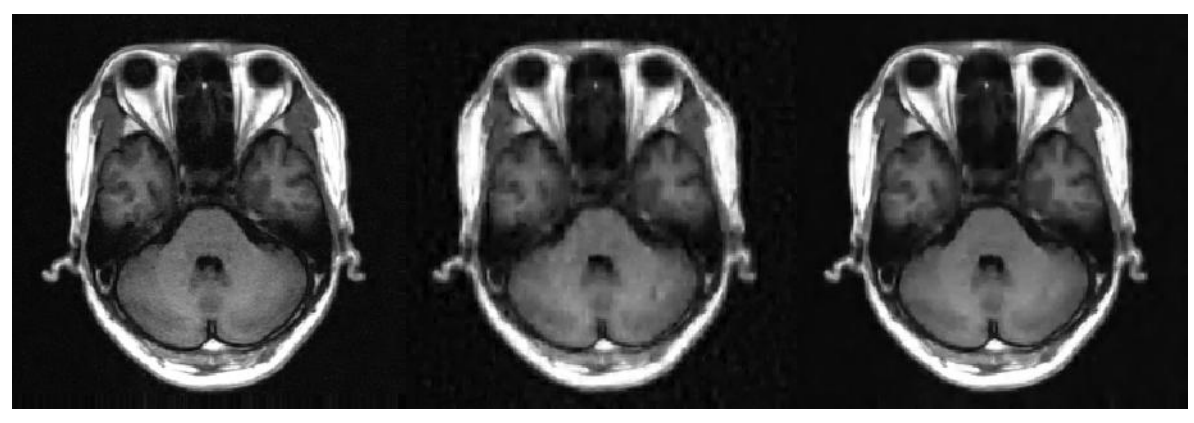

A

B

$\mathrm{C}$

Fig.3 The target image(A), and reconstruction results with $20 \%$ of fully sampled data by conventional CS-MRI (B) and proposed method for 4-level decomposition (C)

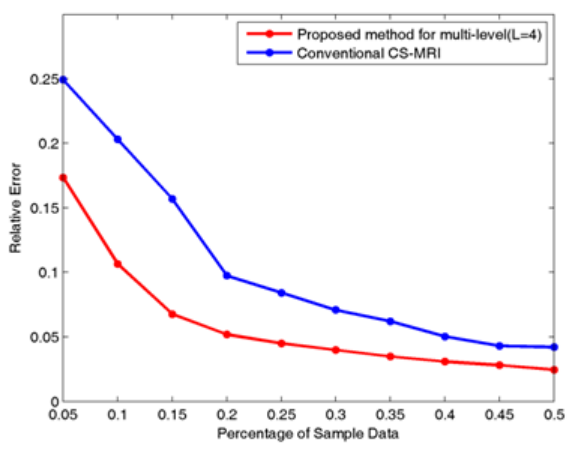

A

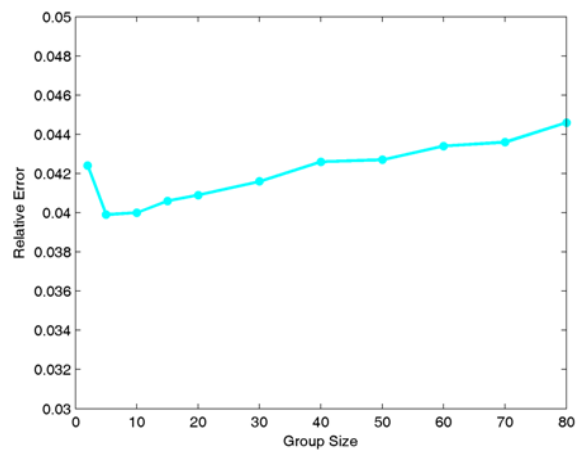

$\mathrm{B}$

Fig.4 Relative errors of reconstructed images by different methods (A), relative error with different group sizes through the proposed method (B)

Through many simulations, we find that, besides MR image, the proposed method can also be applied to other types of images. Different types of images have different energy distributions, so the cardinal of the known support set and the optimal group size can be different. For natural images, such as "camera man", the recovery performance is the best with $20 \%$ largest coefficients and optimal group size 2. How to choose the optimal group size as well as the number of coefficients will be further discussed in our future study.

\section{Conclusion}

We have presented a novel MR reconstruction method incorporating sparsity and structural information for rapid MR imaging. The idea is to reconstruct the image when a part of the support is known and coefficients of the unknown support are 
assigned into even groups. The prior information comes from the reference image. We have showed that the support information and group sparsity of MR images can be exploited to significantly reduce sampling rate or alternatively improve the quality of the reconstructed image.

Acknowledgment This work was supported in part by Natural Science Foundation of China (No.61077022).

\section{References}

1. Babacan SD, Lam F, Peng X, Do MN, Liang ZP (2012). Interventional MRI with sparse sampling using union-of-subspaces. in: IEEE ISBI, 314-317

2. Baraniuk RG, Cevher V, Duarte MF, Hegde C (2010). Model-based compressive sensing. Information Theory, IEEE Transactions on, 56(4), 1982-2001

3. Gamper U, Boesiger P, Kozerke S (2008).Compressed sensing in dynamic MRI. Magn. Reson. Med., 59(2), 365-373

4. Haldar JP, Hernando D, Liang ZP (2011). Compressed-sensing MRI with random encoding. Medical Imaging, IEEE Transactions on, 30(4), 893-903

5. Lu W, Vaswani N (2009). Modified compressive sensing for real-time dynamic MR imaging. in: IEEE ICIP, 3045-3048

6. Lu YM, Do MN (2008). A theory for sampling signals from a union of subspaces. Signal Processing, IEEE Transactions on, 56( 6), 2334-2345

7. Lustig M, Donoho D, Pauly JM (2007). Sparse MRI: The application of compressed sensing for rapid MR imaging. Magn. Reson. Med., 58(6), 1182-1195

8. Prieto, C, Usman, M, Wild, JM, et al. (2012). Group sparse reconstruction using intensitybased clustering. Magn. Reson. Med., 69(4), 1169-1179

9. Qiu C, Vaswani N (2011). Support-predicted Modified-CS for recursive robust principal components' pursuit. in: IEEE ISIT, 668-672

10. Stojnic M, Parvaresh F, Hassibi B (2009). On the reconstruction of block-sparse signals with an optimal number of measurements. Signal Processing, IEEE Transactions on, 57(8), 30753085

11. Trzasko J, Manduca A (2009). Highly undersampled magnetic resonance image reconstruction via homotopic $l_{0}$-minimization. Medical Imaging, IEEE Transactions on, 28(1), 106-121

12. Usman M, Prieto C, Schaeffter T, Batchelor PG (2011). K-t group sparse: A method for accelerating dynamic MRI. Magn. Reson. Med., 62(3), 1163-1176

13. Vaswani N, Lu W (2010). Modified-CS: Modifying compressive sensing for problems with partially known support. Signal Processing, IEEE Transactions on, 58(9), 4595-4607 